Rev. SINAPSIS, Vol. 5, No 2, Diciembre 2014

ISSN $1390-7832$

\title{
Diagnóstico y clasificación de los reactivos peligrosos utilizados en el laboratorio de bromatología de la UNESUM
}

\section{Los reactivos peligrosos usados en laboratorios}

Carlos Julio Cañarte Bello. Mg. Sc ${ }^{(1)}$

Patricia López Loor Mg. Sc. ${ }^{(2)}$

Carlos Suárez Campozano . Mg Sc ${ }^{(3)}$

Gilberto Pinto Pinto Mg.Sc ${ }^{(4)}$

${ }^{(1)}$ Universidad Estatal del Sur de Manabí, Ecuador

(2)Universidad Estatal del Sur de Manabí, Ecuador

${ }^{(3)}$ Universidad Estatal del Sur de Manabí, Ecuador

(4)Universidad Estatal del Sur de Manabí, Ecuador

Contacto: ingagrcjcb@hotmail.com

Receptado: 03/07/2014 Aceptado: 04/09/2014

\section{Resumen}

El estudio se basó en realizar un diagnostico y clasificación de los reactivos peligrosos utilizados y almacenados, durante el periodo académico del segundo semestre (2007) en que se inician las actividades de investigación y docencia hasta el primer semestre (2014), en las áreas del laboratorio de bromatología. Se estableció un formulario para la revisión de los inventarios existentes sobre las sustancias químicas almacenadas y utilizadas en el laboratorio, proponer la implantación de un manejo seguro aplicando la ley de Gestión Ambiental de Ecuador en las operaciones de docencia e investigación, la obtención de estos datos permitió la clasificación de los reactivos de acuerdo con el Sistema Globalmente Armonizado de la ONU; correspondientemente al $\mathrm{N}^{\circ}$. Código de ONU, niveles de riesgos, clases de riesgos, clase de Peligrosidad y la cantidad de todos los reactivos peligrosos almacenados y utilizados en el laboratorio. Los resultados sobre la clasificación de los reactivos químicos, permitió determinar los reactivos de mayor o menor uso en el laboratorio. El apropiado manejo de los reactivos peligrosos, con su correspondiente clasificación basada en los niveles de riesgos, clases de riesgos y clase de peligrosidad como la base primordial para la implantación de un sistema de aseguramiento de calidad de la gestión ambiental en los laboratorios de nuestra Alma Mater. De 
Rev. SINAPSIS, Vol. 5, No 2, Diciembre 2014

ISSN $1390-7832$

hecho la clasificación de los reactivos peligrosos permite minimizar costos económicos en el desarrollo de las investigaciones y la aplicación de prácticas de trabajo educativas por parte de los docentes estudiantes y funcionarios de apoyo durante la manipulación de estos reactivos químicos.

Palabras clave: costos económicos, niveles de riesgos, clases de riesgos, clase de peligrosidad, reactivos peligrosos, sistema de aseguramiento de calidad de la gestión ambiental

\title{
Diagnosis and classification of hazardous reagents used in the laboratory of Food Science UNESUM
}

\begin{abstract}
The study was based on an inventory or diagnosis, of dangerous reagents used and stored, during the second half of the academic period (2007), when researching and teaching activities are stared, until first half (2014), in the areas of food science lab. A form for the review of existing inventories of chemicals stored and used in the laboratory was established to propose the introduction of a safe handling, applying the Environment Management Ecuadorian Law, in teaching and research operations, obtaining these data allowed the classification of reagents according to the Globally Harmonized System of the United Nations, levels and types of risks, the amount of all stored hazardous reagents used in the laboratory. The results on the classification of the chemical reagents, made possible the determination of the class of reagents of varying laboratory use. The proper management of hazardous reagents, with their classification based on risk levels and types of risks as the primary basis for the implementation of a quality assurance system of environmental management in the laboratories of our Alma Mater. In fact, the classification of hazardous reagents minimizes economic costs in the development of research and practical application of educational work by students and teachers and support staff when handling these chemical reagents.
\end{abstract}

Key words: economic costs, levels of risk, types of risk, hazardous reagents, quality assurance system of environmental management. 
Rev. SINAPSIS, Vol. 5, No 2, Diciembre 2014

ISSN 1390 - 7832

\section{Introducción}

Los reactivos peligrosos, para su manejo, están sujetos, actualmente, a normas legales, como el Acuerdo Ministerial $\mathrm{N}^{\circ} 161$, expedido por el Ministerio del Ambiente de Ecuador, con el propósito de reglamentar las actividades que realizan las instituciones de salud, industrias, laboratorios, centros de educación; así como los de Educación Superior, de investigación y experimentación.

Conviene entonces, indicar que cada institución debe reglamentar el manejo de los reactivos peligrosos, con el firme propósito de identificar y clasificar los productos tóxicos por categorías y características específicas; además, de establecer políticas de clasificación, manejo, disposición transitoria y final de los desechos tóxicos generados en las actividades de investigación y docencia; además, es indispensable contar con una infraestructura que facilite tomar las acciones necesarias.

Se debe, entonces, considerar una apropiada gestión que observe los procesos de generación, manipulación, acondicionamiento, almacenamiento y tratamiento final, a bajo costo económico y procurando causar la menor complicación o efecto negativo al medio ambiente.

En cumplimiento a la Ley Orgánica de Salud, (2006), La exposición a los productos químicos puede causar o contribuir efectos serios sobre la salud tales como enfermedades del corazón, lesiones a los riñones y a los pulmones, esterilidad, cáncer, quemaduras y erupciones. Algunos productos químicos pueden presentar también riesgos para la seguridad y presentan el potencial de causar incendios, explosiones y demás accidentes de envergadura.

Debido a la seriedad de estos problemas de salud y seguridad, la Administración de la Salud y Seguridad Ocupacional (OSHA) ha establecido una norma denominada "Información sobre Riesgos". Su propósito principal es el de asegurar que los empleadores y empleados conozcan sobre los riesgos laborales y cómo protegerse contra estos; Esto habrá de reducir la incidencia de las enfermedades y lesiones causadas por los productos peligrosos que ocasionan la muerte, lesiones graves o efectos desfavorables a la salud y el medio ambiente. Algunos productos químicos pueden presentarse de manera potencial, provocar incendios, explosiones y demás accidentes de envergadura. OSHA 3117, (1989). 
Rev. SINAPSIS, Vol. 5, No 2, Diciembre 2014

La Universidad Estatal del Sur de Manabí (UNESUM), Ecuador, Provincia de Manabí, Cantón Jipijapa, tiene la responsabilidad de cumplir con las normas legales reglamentarias vigentes en la Ley de Gestión Ambiental de Ecuador, sobre los reactivos químicos tóxicos, por lo que es obligatorio que los laboratorios de docencia e investigación, dentro de las cuales se manejan y se efectúan actividades que generan residuos tóxicos que representan un grave peligro para la salud de los que manipulan y en general, al medio ambiente, se aplique la ley en vigencia. Si bien, el volumen de los reactivos utilizados en el laboratorio de bromatología es pequeño, no dejan de ser importantes las características de peligrosidad que reflejan estos reactivos químicos tóxicos, por esta razón es imperativo la implantación de un sistema de aseguramiento de calidad de la gestión ambiental en los laboratorios de nuestra Alma Mater.

En la revisión bibliográfica no se ha encontrado artículos relacionados con el tema en el Ecuador, sin embargo en Costa Rica la Universidad Nacional registra un trabajo de investigación denominado: Clasificación de reactivos químicos en los laboratorios, Mora B. (2012).

La presente investigación se justifica, al no encontrar antecedentes sobre el uso y manejo de reactivos químicos en este centro de estudios superior; por la inexistencia de un procedimiento de manejo seguro que conozcan y apliquen los usuarios, es necesario realizar el diagnóstico de los reactivos que se manipulan en el laboratorio de bromatología, desde el periodo académico del segundo semestre del 2007, en que se inician las actividades de investigación y docencia hasta el primer semestre del año 2014.

El objetivo de este trabajo fue establecer un diagnóstico dentro del laboratorio de bromatología aplicando la ley de Gestión Ambiental de Ecuador en las operaciones de docencia e investigación, a partir de la clasificación de sus reactivos químicos tóxicos que causen impactos ambientales, que resultan de los procesos de análisis en el laboratorio de bromatología de la Universidad Estatal del Sur de Manabí.

La metodología se orienta a realizar un diagnóstico inicial sobre la identificación y clasificación de los reactivos peligrosos en las diferentes áreas del laboratorio de bromatología de la Universidad Estatal del Sur de Manabí.

\section{Materiales y métodos}


Rev. SINAPSIS, Vol. 5, No 2, Diciembre 2014

ISSN 1390 - 7832

Para llevar a cabo el estudio de las sustancias químicas en el laboratorio de bromatología de la UNESUM, se enfocó en el uso de los reactivos, tanto para la docencia como para la investigación, en existencia y de uso común relacionados con el estudio y análisis de alimentos como parte del pensum de estudio y entrenamiento de los estudiantes de las carreras de ingeniería agropecuaria, medio ambiente, forestal, administración de empresas agropecuarias, licenciatura en laboratorio clínico, enfermería, ecoturismo y actividades de investigación que llevan a cabo investigadores de esta casa de estudio. La población inicial del estudio (reactivos del laboratorio en existencia) se estableció considerando aquellos utilizados a partir del segundo semestre (2007) y primer semestre (2014).

Para realizar el diagnóstico, de los reactivos peligrosos utilizados y almacenados, se realizó la clasificación, con base en la búsqueda bibliográfica y también, en tablas de seguridad (Safety data), provista en los recipientes y manuales de laboratorio sobre los reactivos y residuos químicos considerando sus características, su clasificación, su reactividad, toxicidad y peligrosidad.

Martínez, et al (2005), en la guía para la gestión integral de residuos peligrosos presenta las características, simbología, niveles de riesgos de los reactivos peligrosos utilizados y almacenados, conforme a la definición de las Naciones Unidas, para el manejo de sustancias químicas.

Se utilizó como referencia el Sistema Globalmente Armonizado de la ONU. (GHS, Global Harmonized System) es el sistema que clasifica los productos químicos de acuerdo con tres criterios: Riesgos físicos, Riesgos para la salud y Riesgos para el medio ambiente. Cada uno de estos grupos se subdivide en diferentes categorías (Universidad de Brasilia, 2008).

En la identificación y clasificación de los materiales y desechos peligrosos, es importante reconocer que existen varias clases o niveles de riesgo, asociados con los diferentes tipos de productos.

Clases de riesgos 
Rev. SINAPSIS, Vol. 5, N², Diciembre 2014

\begin{tabular}{|c|l|}
\hline Clase 1 & Estado sólido, sin equipos especiales \\
\hline Clase 2 & Semisólidos o líquidos. Su riesgo mayor condición fluida \\
\hline Clase 3 & Sólidos o líquidos combustibles en presencia de llama \\
\hline Clase 4 & Sólidos o líquidos explosivos, corrosivos, reactivos o tóxicos \\
\hline Clase 5 & Sólidos, líquidos y gases muy reactivos. Efectos letales \\
\hline
\end{tabular}

Tabla $\mathrm{N}^{\circ}$ 1. Clasificación de riesgos, según establece el Decreto 2635, artículo 8, de la Normativa Ambiental Venezolana.

\begin{tabular}{|l|l|}
\hline \multicolumn{2}{|c|}{ Clase de peligrosidad } \\
\hline Clase 1 & Materias y objetos explosivos \\
\hline Clase 2 & Gases \\
\hline Clase 3 & Líquidos inflamables \\
\hline Clase 4.1 & $\begin{array}{l}\text { Materias solidas inflamables, materias autoreactivas y materias } \\
\text { explosivas desensibilizadas solidas }\end{array}$ \\
\hline Clase 4.2 & \begin{tabular}{l} 
Materias que pueden experimentar inflamación espontánea \\
\hline Clase 4.3
\end{tabular} \\
\hline
\end{tabular}


Rev. SINAPSIS, Vol. 5, N², Diciembre 2014

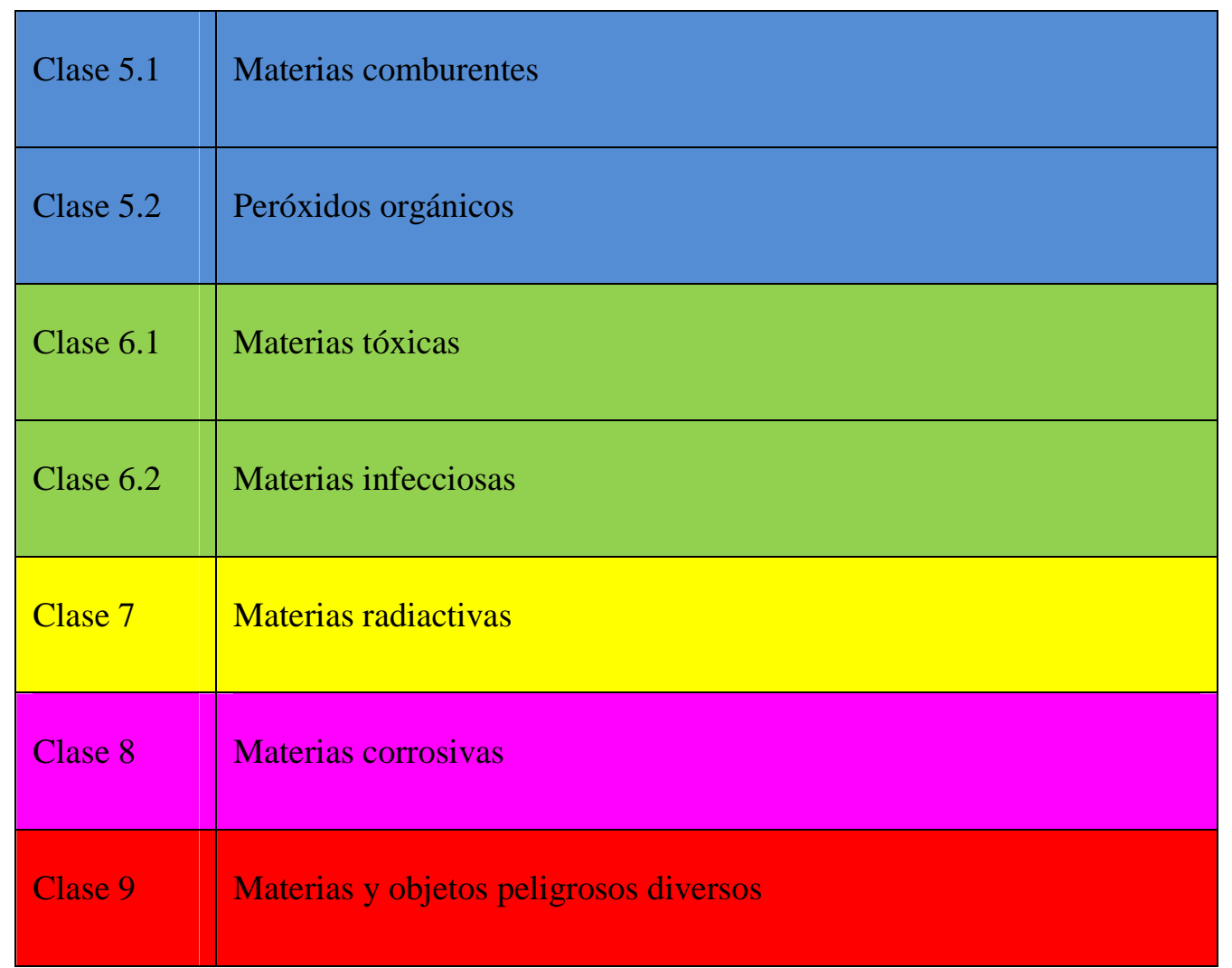

Tabla $N^{\circ}$ 2. Clase de peligrosidad, según ADR, basándose en las recomendaciones del comité de expertos en transporte de mercancías peligrosas.

Al momento de realizar la clasificación de las sustancias, se estableció una tabla que incluye el nombre del reactivo peligroso, Número código ONU, niveles de riesgos ONU, clases de riesgos, la cantidad y clase de peligrosidad según ADR; de todos los reactivos químicos almacenados y utilizados en el período considerado, expresado en la tabla № 3 .

El aspecto metodológico de este trabajo tiene carácter exploratorio, analítico, participativo y de acción. Se desarrolló en varias etapas. Este análisis permitió identificar las sustancias químicas que se manejan en las áreas del laboratorio de bromatología, a partir de cada uno de los caracteres identificados, dada la complejidad y frecuencia de las actividades.

\section{Resultados}

A continuación se presentan de manera tabulada, las características de peligrosidad de los reactivos químicos, existentes en el laboratorio de bromatología en el ciclo contemplado, de 
Rev. SINAPSIS, Vol. 5, No 2, Diciembre 2014

acuerdo a los criterios señalados en la metodología, mismos que se reflejan en la clasificación de la siguiente tabla:

\begin{tabular}{|c|c|c|c|c|c|}
\hline Reactivos Peligrosos & $\begin{array}{c}\text { No. Código } \\
\text { de ONU }\end{array}$ & Niveles de & Clases de & Cantidad & $\begin{array}{c}\text { Clase de } \\
\text { Peligrosidad }\end{array}$ \\
\hline Acetonitrilo & 1648 & H3-H6.1 & $2-3-0$ & $8 \mathrm{~L}$ & 3 \\
\hline Acetato de Zinc Dihidratado & 3077 & H6.1-H12 & $2-1-0$ & $500 \mathrm{~g}$ & 3 \\
\hline Ácido Acético grado Analítico & 2789 & $\mathrm{H} 3-\mathrm{H} 8$ & $3-2-0$ & $25 \mathrm{~L}$ & 8 \\
\hline Ácido Bórico & N/D & H6.1 & $1-0-0$ & $500 \mathrm{~g}$ & 9 \\
\hline Ácido Clorhídrico & 1789 & H8 & $3-0-1$ & $10 \mathrm{~L}$ & 8 \\
\hline Ácido Nítrico & 2031 & H3-H5.1-H8 & $3-0-0$ & $5 \mathrm{~L}$ & 8 \\
\hline Ácido Ortofosfórico $85 \%$ & 1805 & H8-H12 & $3-0-1$ & $25 \mathrm{~L}$ & 8 \\
\hline Ácido Sulfúrico & 1830 & $\mathrm{H} 8$ & $3-0-2$ & $72.5 \mathrm{~L}$ & 8 \\
\hline Almidón & $\mathrm{N} / \mathrm{D}$ & H6.1 & $0-1-0$ & $250 \mathrm{~g}$ & N/A \\
\hline Anaranjado de Metilo & 2811 & H6.1 & $2-1-0$ & $100 \mathrm{~g}$ & 3 \\
\hline Azul de Metileno & $\mathrm{N} / \mathrm{D}$ & H4.1-H6.1 & $1-1-0$ & $25 \mathrm{~g}$ & N/A \\
\hline
\end{tabular}


ISSN $1390-7832$

Rev. SINAPSIS, Vol. 5, N² 2, Diciembre 2014

\begin{tabular}{|c|c|c|c|c|c|}
\hline Bicarbonato de Sodio & N/D & H6.1 & $1-0-0$ & $6 \mathrm{~kg}$ & 6.1 \\
\hline Cloroformo & 1888 & H6.1 & $2-0-0$ & $1 \mathrm{~L}$ & 6.1 \\
\hline Cloruro de Calcio & N/D & H6.1 & $1-0-0$ & $500 \mathrm{~g}$ & 6.1 \\
\hline Cloruro de Sodio & N/D & $\mathrm{H} 8$ & $1-0-0$ & $1 \mathrm{~kg}$ & 6.1 \\
\hline Cromato de Potasio & 3288 & H6.1-H12 & $3-0-1$ & $250 \mathrm{~g}$ & 6.1 \\
\hline Etanol & 1170 & $\mathrm{H} 3$ & $2-3-1$ & $8 \mathrm{~L}$ & 3 \\
\hline Eter Dietílico & 1155 & H3-H6.1 & $1-4-1$ & $33 \mathrm{~L}$ & 3 \\
\hline Eter de Petróleo & 1268 & H3-H6.1-H12 & $1-4-0$ & $34 \mathrm{~L}$ & 3 \\
\hline Ftalato de Potasio & N/D & H8 & $1-1-0$ & $500 \mathrm{~g}$ & N/A \\
\hline Ftalaldehído & 2923 & H6.1-H8- H12 & & $10 \mathrm{~g}$ & 6.1 \\
\hline Fenolftaleina & N/D & H6.1 & $1-1-0$ & $500 \mathrm{~g}$ & N/A \\
\hline Hidróxido de Amonio & 2672 & H3-H6.1-H8 & $3-1-0$ & $5 \mathrm{~L}$ & 2 \\
\hline Hidróxido de Sodio $98 \%$ & 1823 & H8 & $4-0-2$ & $10 \mathrm{Kg}$ & 8 \\
\hline Hidróxido de Sodio al 1N & 1824 & H8 & $3-0-0$ & $10 \mathrm{~L}$ & 8 \\
\hline Hidróxido de Sodio al 0.1N & 1824 & $\mathrm{H} 8$ & $3-0-0$ & $10 \mathrm{~L}$ & 8 \\
\hline Hidróxido de Potasio & 1813 & H6.1-H8 & $3-0-1$ & $1 \mathrm{~kg}$ & 8 \\
\hline
\end{tabular}


Rev. SINAPSIS, Vol. 5, No 2, Diciembre 2014

\begin{tabular}{|c|c|c|c|c|c|}
\hline Metanol & 1230 & H3-H6.1 & $2-3-0$ & $8 \mathrm{~L}$ & 3 \\
\hline Negro de Eriocromo $T$ & $\mathrm{~N} / \mathrm{D}$ & H6.1-H12 & $1-0-0$ & $25 \mathrm{~g}$ & 6.1 \\
\hline N. Hexano & 1208 & H3-H6.1-H11 & $1-3-0$ & $4 \mathrm{~L}$ & 3 \\
\hline Nitrato de Plata & 1493 & H5.1-H8-H12 & $3-0-0$ & $250 \mathrm{~g}$ & 5.1 \\
\hline $\begin{array}{l}\text { Purpurato de amonio } \\
\text { (Murexida) }\end{array}$ & N/D & H6.1 & $1-0-0$ & $5 \mathrm{~g}$ & 6.1 \\
\hline Rojo de Metilo & N/D & H4.1-H8-H12 & $2-3-1$ & $100 \mathrm{~g}$ & 9 \\
\hline Sulfato de Cobre & $\mathrm{N} / \mathrm{D}$ & H6.1-H12 & $2-0-0$ & $1 \mathrm{~kg}$ & 9 \\
\hline Sulfato de Sodio Anhidro & $\mathrm{N} / \mathrm{D}$ & H6.1 & $1-0-1$ & $5 \mathrm{~kg}$ & N/A \\
\hline Sulfato de Zinc & N/D & H6.1-H8-H12 & $1-0-1$ & $1 \mathrm{~kg}$ & N/A \\
\hline Tartrato de Potasio y Sodio & $\mathrm{N} / \mathrm{D}$ & H6.1-H8 & $1-1-0$ & $1 \mathrm{~kg}$ & 6.1 \\
\hline Tiosulfato de Sodio & N/D & H6.1-H12 & $1-0-1$ & $500 \mathrm{~g}$ & N/A \\
\hline Yoduro de Potasio & N/D & H6.1 & $1-0-0$ & $1 \mathrm{~kg}$ & N/A \\
\hline Zinc Granalla & 1436 & H3-H12 & $1-1-1$ & $1 \mathrm{~kg}$ & 4.3 \\
\hline
\end{tabular}

Tabla $\mathrm{N}^{\circ}$ 3. Clasificación de los reactivos peligrosos utilizados en las diferentes áreas del laboratorio de bromatología de la Universidad Estatal del Sur de Manabí.

Con relación a la tabla anterior, y en lo referente a la columna: $\mathrm{N}^{\circ}$. Código de ONU, son números de cuatro dígitos, usados para identificar sustancias o materiales peligrosos (explosivos, líquidos flamables, sustancias toxicas, etc.) en el marco del transporte internacional; Niveles de 
Rev. SINAPSIS, Vol. 5, No 2, Diciembre 2014

ISSN 1390 - 7832

Riesgos: criterio utilizado en la clasificación y etiquetado de productos químicos; clases de riesgos los tres números significan: el primer número riesgos a la salud, segundo número inflamabilidad y tercer número corresponde a la reactividad, respectivamente en su orden; clase de peligrosidad, se refiere al código internacional de transporte.

El estudio permitió obtener la información sobre la cantidad de reactivos almacenados y empleados, es importante indicar que al identificar los reactivos peligrosos, como acciones individuales, determinó que en el laboratorio de bromatología, en el periodo 2007 a 2014, se almacena un total de 32.09 kilogramos $(\mathrm{kg})$ de reactivos peligrosos en forma sólida (corrosivo, inflamable y toxico); y $285.5 \mathrm{~L}$ de reactivos químicos en estado líquido (corrosivo, inflamable, toxico y ecotóxicos), como se muestra en la figura 1.

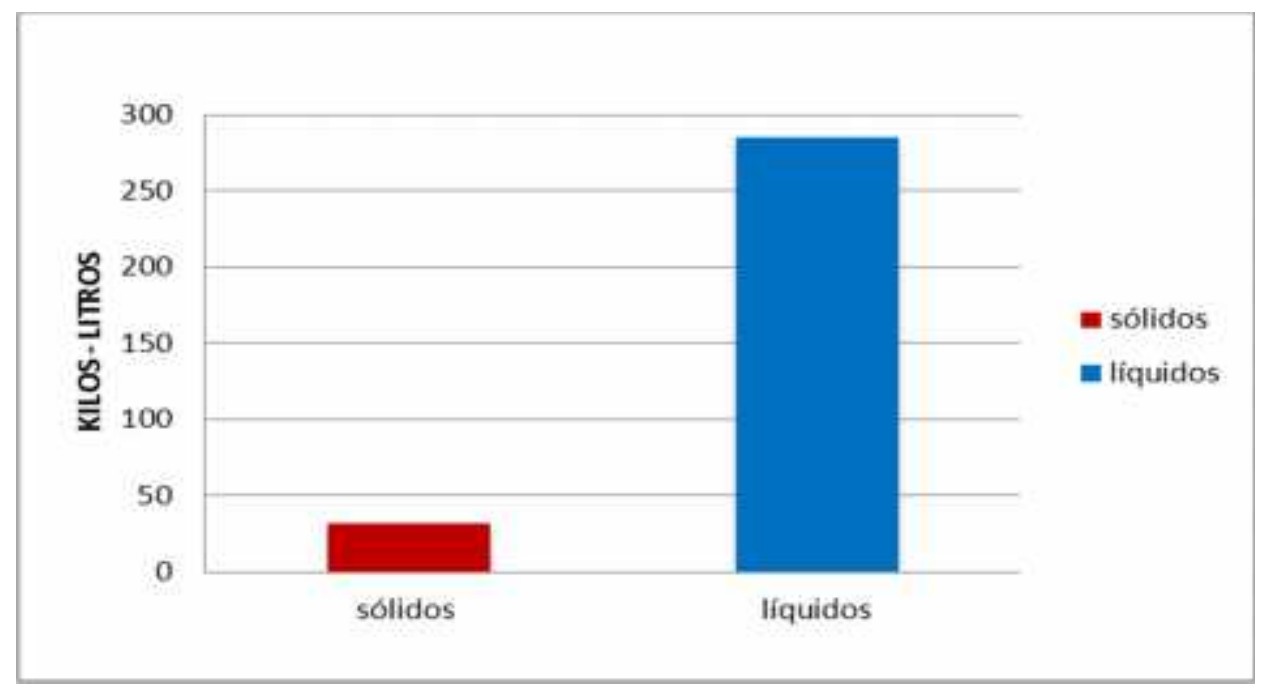

Figura 1. Distribución cuantitativa de reactivos peligrosos en el laboratorio de bromatología

La figura 2, muestra la distribución porcentual de las características de los reactivos peligrosos presentes en el laboratorio de bromatología. Se destacan los reactivos que pertenecen al grupo H6.1 correspondiente al mayor porcentaje presente en este nivel de riesgo a (tóxicos agudos) con el 65\%; el grupo H8 que concierne a (sustancias corrosivas) del nivel de riesgo con el 42.5\%; mientras que el nivel H12 corresponde a (sustancias ecotóxicas) se halla en el 30\%; los niveles H3 con característica de (líquidos inflamables), se encuentra con el 25\%; y en menor porcentaje los niveles H4.1, H5.1 y H11, con características de (solidos inflamables), (sustancias o desechos 
Rev. SINAPSIS, Vol. 5, No 2, Diciembre 2014

ISSN $1390-7832$

oxidantes), (sustancias toxicas con efectos retardados o crónicos) respectivamente del total de reactivos presentes en el laboratorio.

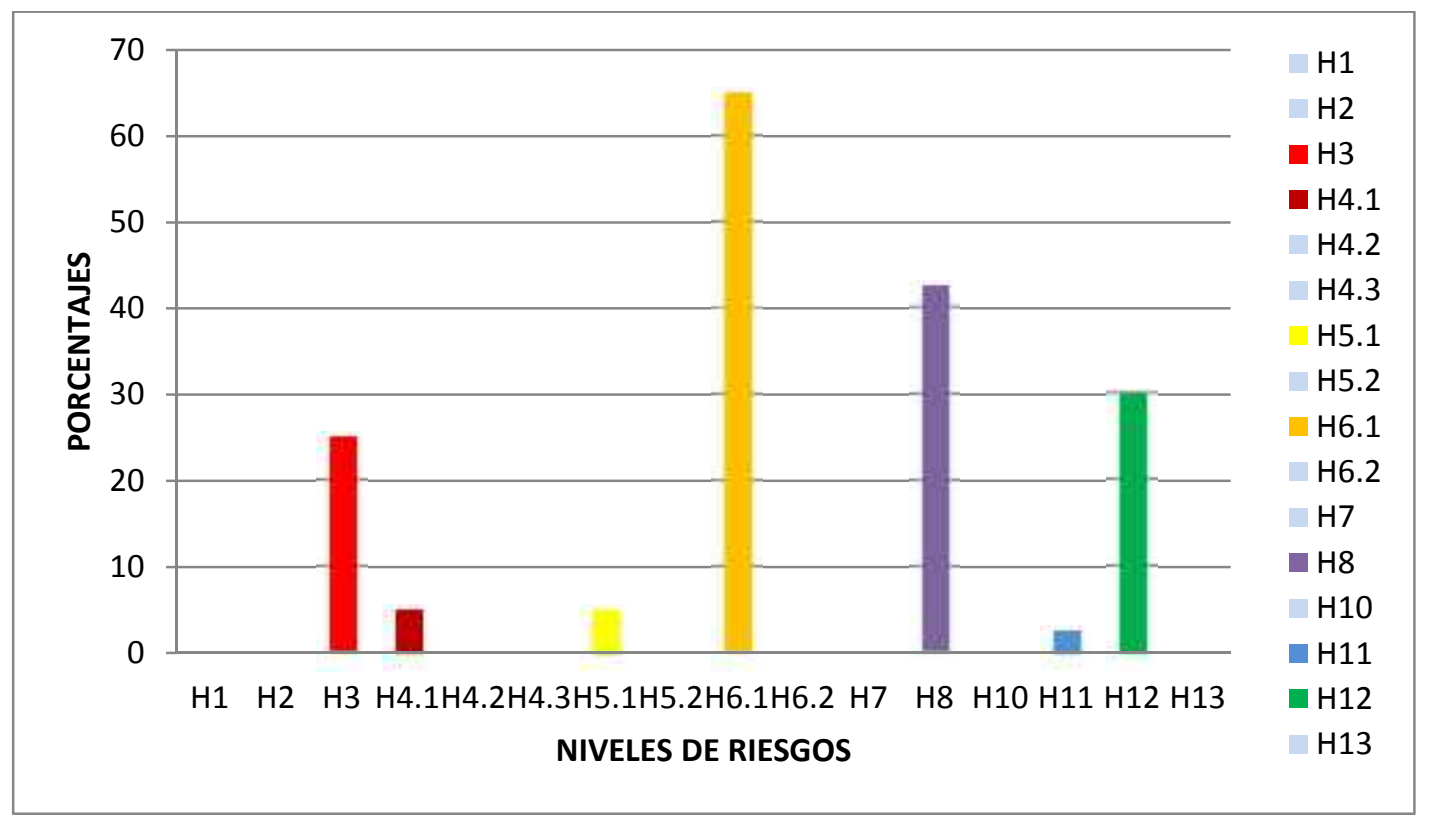

Figura 2. Distribución porcentual de reactivos peligrosos clasificados según niveles de riesgos ONU.

La figura 3, destaca la clase de peligrosidad de los reactivos presentes en el laboratorio de bromatología, con porcentajes del $22.5 \%$ a las clases 6.1 y 8 pertenecientes a materias toxicas y corrosivas individualmente; el $20 \%$ para la clase 3 a líquidos inflamables y productos químicos que no registran peligrosidad, representado en la figura por (N/A); se maneja el 7.5\% de la clase 9 correspondiente a materias y objetos peligrosos diversos; y el 2.5\% para las clase 2 a gases, 4.3 Materias que al contacto con agua desprenden gases inflamables y 5.1 a materias comburentes equitativamente. 
Rev. SINAPSIS, Vol. 5, No 2, Diciembre 2014

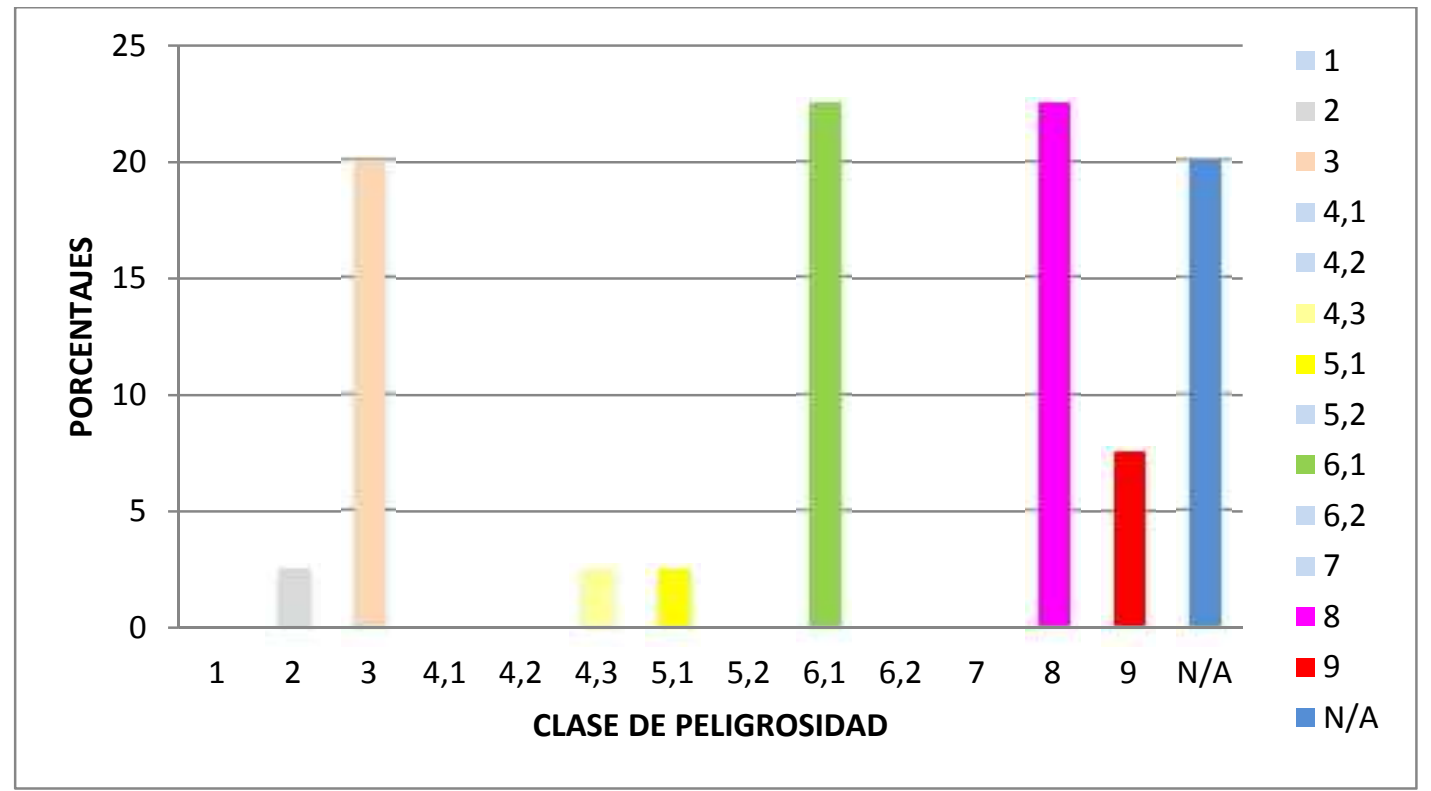

Figura 3. Distribución porcentual correspondiente a clase de peligrosidad clasificada según la ADR.

Producto de la inspección de las áreas de laboratorio se observa, de manera general, la insuficiencia de secciones señalizadas y reservadas para el almacenamiento de reactivos peligrosos o pasivos ambientales. Además, tales reactivos se almacenan en los envases en los que vienen los reactivos originalmente no necesariamente de la misma naturaleza química y otro tipo de materiales, en lugares que no se crearon con este fin.

Se observa que el almacenamiento de los reactivos es completamente inadecuado y en sitios no convenientes, no se dispone de sitio procedente para almacenamiento, tal como se presenta en las gráficas 1.

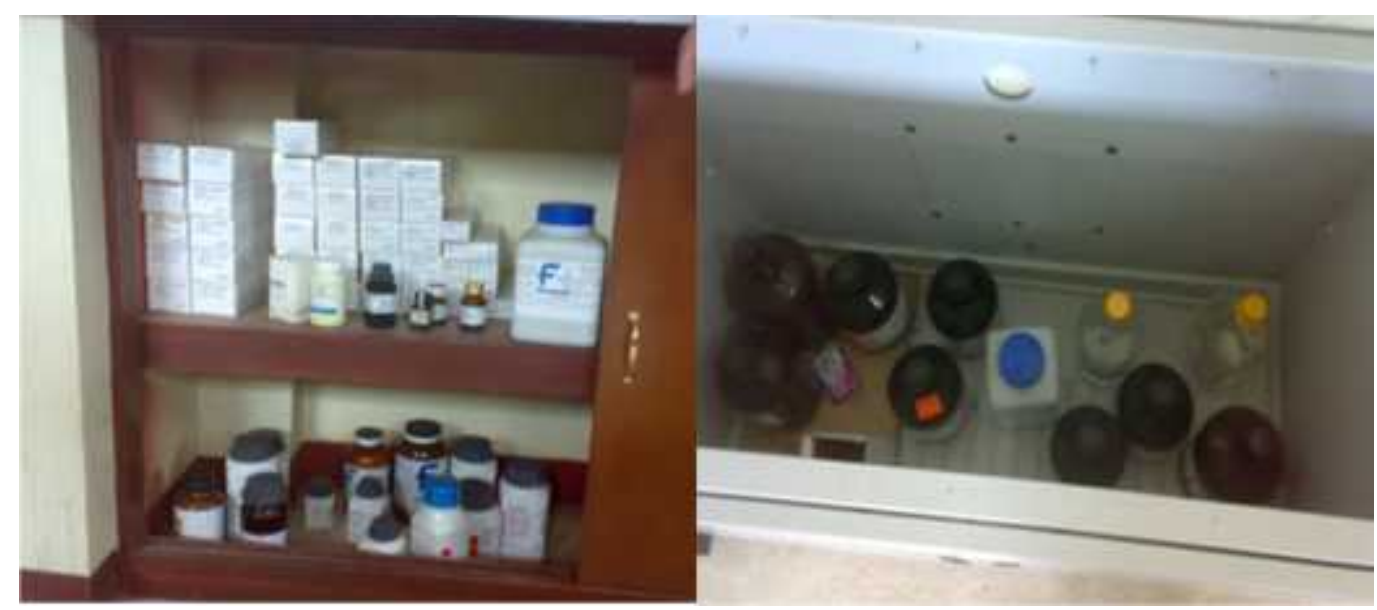


Rev. SINAPSIS, Vol. 5, N² 2, Diciembre 2014

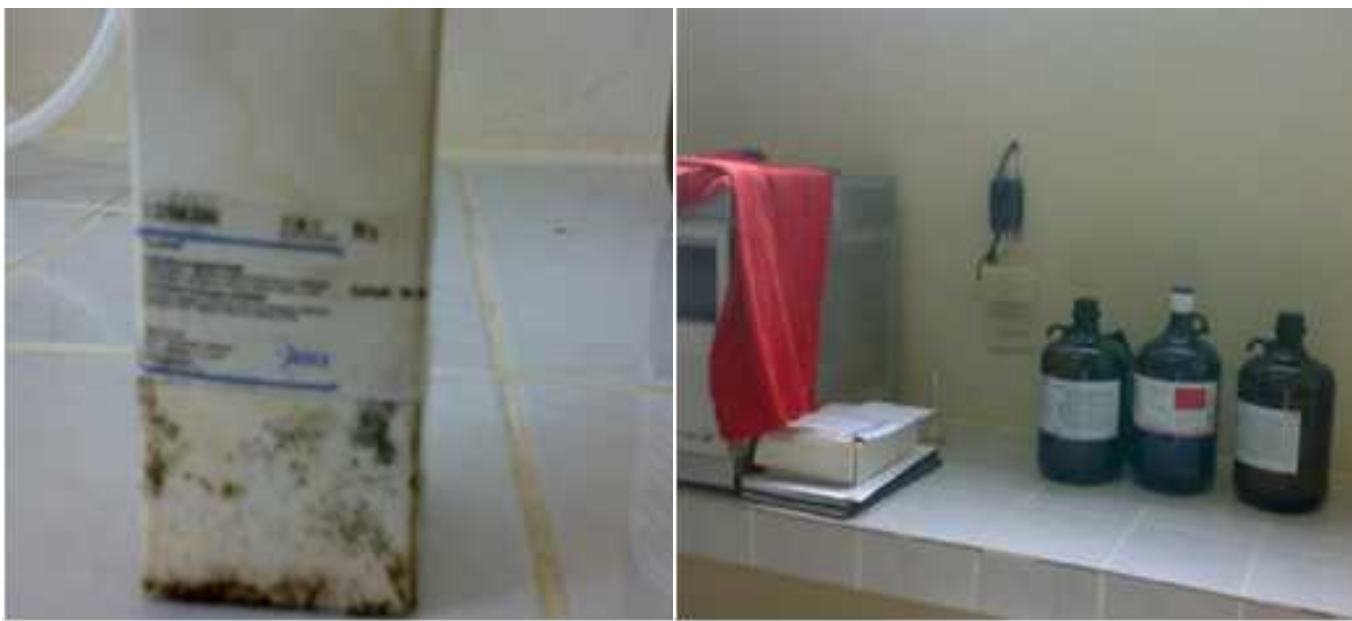

Gráfica 1. Almacenamiento incorrecto de reactivos peligrosos

En la gráfica 2 se puede apreciar el desarrollo de investigaciones y la aplicación de prácticas de trabajo educativas por parte de los docentes, estudiantes y usuarios durante la manipulación de estos reactivos peligrosos, su manejo se torna vulnerable, se aprecia que no se respetan las normas de seguridad, es deficiente la protección del personal.
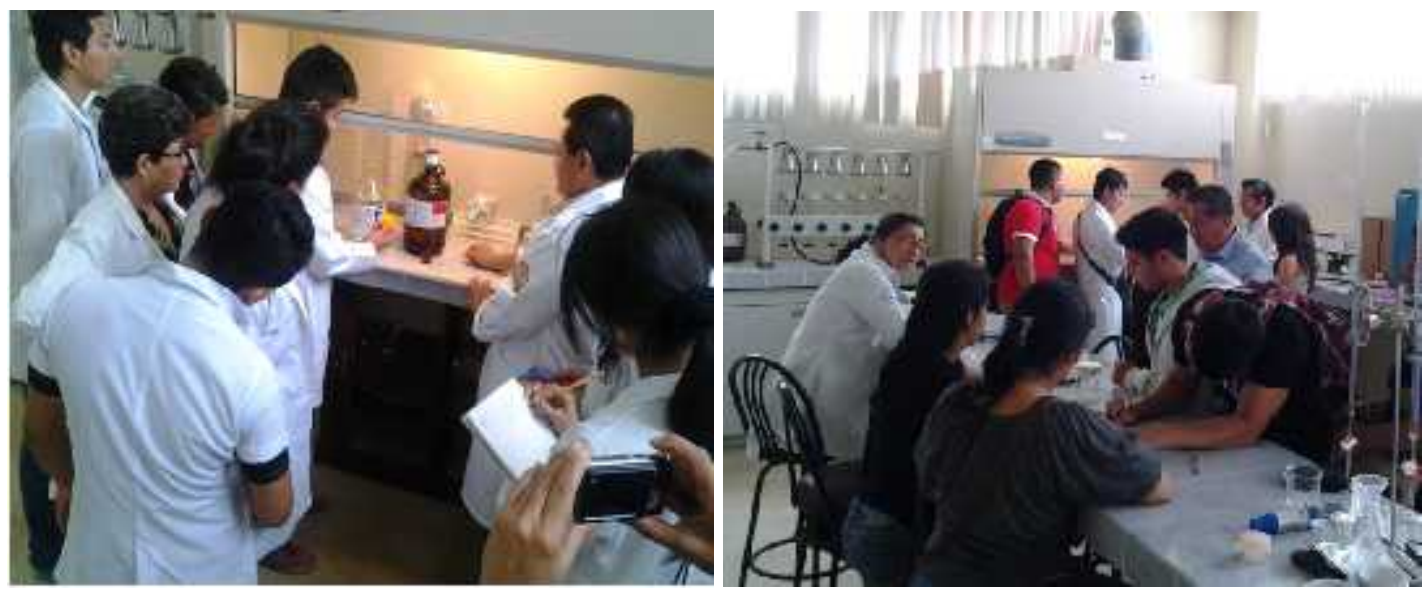

Gráfica 2. La deficiente protección del personal.

\section{Discusión}

Los resultados obtenidos en cuanto al análisis realizado en las áreas del laboratorio de bromatología durante el periodo 2007-2014, establecieron que se ha utilizado una menor cantidad de reactivos sólidos (corrosivo, inflamable y toxico); a diferencia que la mayor cantidad corresponde a los reactivos líquidos (corrosivo, inflamable, toxico y ecotóxicos). 
Rev. SINAPSIS, Vol. 5, No 2, Diciembre 2014

ISSN 1390 - 7832

Además permitió establecer cuales son los reactivos utilizados durante el desarrollo de las actividades de investigación y docencia; a partir de cada una de las características de peligrosidad identificadas, con base al inventario levantado, los resultados determinaron que la mayor cantidad de productos peligrosos presentes, descritos en el diagnóstico concierne a los reactivos que pertenecen al grupo H6.1 correspondiendo este mayor porcentaje al nivel de riesgo (tóxicos agudos) con el 65\%; Se enfatiza de igual manera que los del grupo H8 pertenece a (sustancias corrosivas) en el nivel de riesgo con el 42.5\%; mientras que reactivos peligrosos H12 que corresponde a (sustancias ecotóxicas) arrojo un porcentaje del 30\%; las sustancias H3 con característica de (líquidos inflamables), se encuentra con el 25\%; ya en menor porcentaje se determino reactivos del grupo H4.1, H5.1 y H11, con características de (solidos inflamables), (sustancias o desechos oxidantes), (sustancias toxicas con efectos retardados o crónicos) en su orden. El manejo adecuado de los reactivos químicos y la correspondiente clasificación físicoquímica, basada en el riesgo y peligro de estas sustancias, constituye la base para implementar un ambiente seguro de trabajo en los laboratorios de la Unesum.

Con respecto a la clase de peligrosidad de los reactivos presentes en el laboratorio de bromatología, se obtuvo porcentajes del $22.5 \%$ correspondiéndole a las clases 6.1 y 8 pertenecientes a materias (toxicas y corrosivas) individualmente; el $20 \%$ para la clase 3 a (líquidos inflamables) y productos químicos que no registran peligrosidad, representado en la figura por (N/A); se maneja el 7.5\% de la clase 9 correspondiente a (materias y objetos peligrosos diversos); y en menor presencia el 2.5\% para las clase 2 a (gases), 4.3 (Materias que al contacto con agua desprenden gases inflamables) y 5.1 a (materias comburentes) de manera equivalente.

En los espacios de la UNESUM no se dispone de áreas adecuadas para el almacén de los materiales y desechos peligrosos. No existe un sistema de aseguramiento de calidad para almacenar transitoriamente dichas sustancias en el laboratorio bromatología, tanto para los reactivos utilizados y no utilizados. De igual manera, se observa que el almacenaje de los reactivos es completamente inadecuado y en sitios no convenientes, los espacios destinados a actividades operativas son subutilizadas para ubicar reactivos peligrosos, pues estos deben estar vacíos con la finalidad de evitar accidentes que se pueden generar al estar próximos a conexiones eléctricas, entre otras, tal como se observa en la gráfica 1. 
Rev. SINAPSIS, Vol. 5, No 2, Diciembre 2014

ISSN 1390 - 7832

Por otro lado, se observó el manejo inadecuado de sustancias peligrosas durante el desarrollo de las investigaciones y la aplicación de prácticas de trabajo educativas por parte de los docentes, estudiantes y usuarios, durante la manipulación de estos reactivos químicos, por lo tanto se torna vulnerable para empleadores y empleados cuando los riesgos no son asumido por las autoridades universitarias, esto se manifiesta como una deficiente protección al personal, como se observa en la gráfica 2.

Esta investigación permite tener un cabal conocimiento de la peligrosidad de los reactivos que se manipulan en el laboratorio de bromatología tales como los (tóxicos agudos, sustancias corrosivas, sustancias ecotóxicas, sustancias inflamables). Dependiendo de su concentración y tiempo de exposición los reactivos químicos peligrosos tienen el potencial para causar la muerte, lesiones graves o efectos desfavorables a la salud y el medio ambiente. Algunos productos químicos pueden presentarse de manera potencial, causar incendios, explosiones y demás accidentes de envergadura. Cuando no se aplica la norma denominada "Información sobre Riesgos". Como manifiesta la OSHA 3117.

\section{Conclusión}

A partir del presente trabajo, se logró establecer el diagnóstico y clasificar los reactivos peligrosos utilizados en el laboratorio de bromatología, el mismo que permite conocer el verdadero problema de este espacio al no disponer de áreas adecuadas para el almacenamiento de los materiales y desechos peligrosos. No se maneja un sistema de aseguramiento de calidad para almacenar transitoriamente estas (sustancias tóxicas agudas, corrosivas, inflamables, ecotóxicas), para los reactivos utilizados y no utilizados. De igual forma, se observó que el almacenaje de los reactivos es completamente inadecuado y en sitios no convenientes, los espacios destinados a actividades operativas son utilizadas para ubicar reactivos peligrosos, otro de los problemas es el manejo inadecuado de sustancias peligrosas durante el desarrollo de las investigaciones y la aplicación de prácticas de trabajo educativas por parte de los docentes, estudiantes y usuarios, se torna peligroso para empleadores y empleados cuando los riesgos se manifiestan como una deficiente protección al personal. 
Rev. SINAPSIS, Vol. 5, No 2, Diciembre 2014

ISSN 1390 - 7832

Además los aparatos extintores que existen no son los apropiados para extinguir cualquier connato de incendio, por lo que frente al riesgo y peligro que estas sustancias instauran no se debe dispone del equipo preventivo adecuado.

Conviene, entonces, sentar las bases para la implantación de un manejo seguro aplicando la ley de Gestión Ambiental de Ecuador en las operaciones de docencia e investigación, para el alcance de la eficiencia considerando las valoraciones correspondientes para la defensa del ambiente, el hombre y la economía de los recursos, en el desarrollo de los procesos. Se sugiere implantar inspecciones en cada una de las operaciones con el propósito de normalizar los procedimientos.

\section{Bibliografía}

1. Martínez Javier, Mallo Marisol, Álvarez Jacqueline, Salvarrey Ana, Gristo Pablo, (2005), Guía para la gestión integral de residuos peligrosos, Fundamentos, Tomo I, Centro coordinador del convenio de Basilea para América Latina y el Caribe, Montevideo, Uruguay.

2. Mora B. José C., Piedra M. Gilberto, Benavides R. David, Ruepert R. Clemens, (2012), Clasificación de reactivos químicos en los laboratorios de la Universidad Nacional, Tecnología en Marcha, Vol. 25, N 3, Costa Rica.

3. Registro Oficial $N^{\circ} 725$, (2002), Legislación ambiental secundaria, Edición Especial, 16 de noviembre.

4. Registro Oficial Suplemento No 423, (2006), Ley Orgánica de Salud, libro II, Salud y Seguridad Ambiental, Titulo Único, Capitulo II, de los desechos comunes, infecciosos, especiales y de las radiaciones ionizantes y no ionizantes, (Arts. 103, 104), 22 de diciembre.

5. Registro Oficial No 1, (2008), Constitución política de la Republica del Ecuador. Capitulo II derechos del buen vivir, sección segunda, (Art. 14, 15, 71, 72), 19 de octubre.

6. Registro Oficial Suplemento del $\mathrm{N}^{\circ} 631$, (2012) Ministerio del Ambiente, Acuerdo Ministerial $N^{\circ} 161$, Reglamento para la Prevención y Control de la Contaminación por Sustancias Químicas Peligrosas, Desechos Peligrosos y Especiales, del 1 de febrero.

7. OSHA 3117, (1989). Información sobre los riesgos de productos químicos.

8. Universidad de Brasilia. (2008). International Symposium on Residue Management in Universities. Brasil. 